\title{
The Combination PARP Inhibitor Olaparib With Temozolomide in an Experimental Glioblastoma Model
}

\author{
KIHWAN HWANG ${ }^{1}$, JIN-HAN LEE ${ }^{2}$, SANG HO KIM ${ }^{3}$, KYEONG-O GO ${ }^{4}$, \\ SO YOUNG JI ${ }^{1}$, JUNG HO HAN ${ }^{1}$ and CHAE-YONG KIM ${ }^{1,5}$ \\ ${ }^{1}$ Department of Neurosurgery, Seoul National University Bundang Hospital, Seongnam-si, Republic of Korea; \\ ${ }^{2}$ Seoul National University College of Medicine, Seoul, Republic of Korea; \\ ${ }^{3}$ School of Medicine, The University of Auckland, Auckland, New Zealand; \\ ${ }^{4}$ Department of Neurosurgery, Gyeongsang National University Hospital, Gyengsangnam-do, Republic of Korea; \\ ${ }^{5}$ Department of Neurosurgery, Seoul National University College of Medicine, Seoul, Republic of Korea
}

\begin{abstract}
Background/Aim: Poly (ADP-ribose) polymerase (PARP) inhibition could enhance the efficacy of temozolomide and prolong survival in patients with glioblastoma. The aim of this study was to evaluate the combination of the PARP inhibitor olaparib with temozolomide in the treatment of glioblastoma. Materials and Methods: The in vitro and in vivo antitumor effects of the PARP inhibitor olaparib together with temozolomide were evaluated. The in vitro experimental glioblastoma model involved $O^{6}$-methylguanine methyltransferase (MGMT) promoter-methylated (U87MG, U251MG) and MGMT promoter-unmethylated (T98G) glioblastoma cell lines using In this model cell viability and apoptosis were assessed. For the in vivo studies, nude mice bearing orthotopically xenografted glioblastoma cell lines (U87MG) were randomized to four experimental groups: i) the untreated, ii) temozolomide alone, iii) olaparib alone and iv) olaparib and temozolomide combination groups. Mice were treated daily for 4 weeks and monitored for tumor growth and survival. Results: In vitro we found that the combination of olaparib with temozolomide enhanced temozolomideinduced cytotoxicity in all glioblastoma cell lines regardless of the status of MGMT promoter methylation. In vivo, mice treated with temozolomide alone or in combination with
\end{abstract}

This article is freely accessible online.

Correspondence to: Chae-Yong Kim, Department of Neurosurgery, Seoul National University Bundang Hospital, 82, Gumi-ro 173 Beon-gil, Bundang-gu, Seongnam-si, Gyeonggi-do 13620, Republic of Korea. Tel: +82 317877165, Fax: +82 317874097, e-mail: chaeyong@snu.ac.kr

Key Words: Glioblastoma, olaparib, temozolomide, combination chemotherapy. olaparib showed greater survival than those untreated or with the olaparib monotherapy, as well as significantly decreased tumor volume. There was no significant difference in survival and tumor volume between temozolomide alone and the combination treatment. Conclusion: The combination of the PARP inhibitor olaparib with temozolomide could be promising candidates for combination therapy of glioblastoma regardless of the MGMT promoter methylation status.

Glioblastoma is the most common and deadliest glioma subtype in adults, with an age-adjusted incidence of 0.59 to 3.69 per 100,000 persons (1). Glioblastoma has a highly aggressive behavior, and, without any treatment, its median overall survival (OS) is limited to approximately 3 to 6 months. The standard care includes maximal safe resection, followed by concurrent chemoradiotherapy with temozolomide (TMZ) and subsequent treatment with adjuvant temozolomide, which is named Stupp's protocol (2).

Although patients with glioblastoma are treated multimodally, the prognosis remains poor. The median OS is still limited to 14.6 months, while the OS rate after 2 years is $27 \%$, dropping down to $3 \%$ to $7 \%$ after 5 years $(2,3)$. Additionally, almost all glioblastoma patients' relapse, and over $86 \%$ of their recurrences are in the irradiation field (4). The difficulty of treating glioblastoma is due to several complicating factors. As glioblastoma is a brain tumor, treatments are restricted by the blood-brain barrier (BBB) and by the risks it bears due to the uniqueness of the brain tissue, profound susceptibility to interstitial chemotherapy and limited repair capacity (5). In addition, glioblastoma cells are considered chemo- and radioresistant $(2,6,7)$.

The resistance to treatment could be explained by the presence of glioblastoma stem cells (GSCs). GSCs are a specific subpopulation of glioblastoma cells with characteristics of tumor stem cells, such as self-renewal, 
differentiation, metastatic abilities, unlimited proficiency, and high DNA repair capacity $(6,7)$. Among the properties of GSCs, their high DNA repair capacity is widely believed to participate in their resistance against chemotherapy and radiotherapy. In GSCs, proteins that respond to DNA damage, such as checkpoint kinase 1 (CHK1), ATR serine/threonine kinase (ATR), ATM serine/threonine kinase (ATM), and poly (ADP-ribose) polymerase 1 (PARP1), are upregulated $(6,8)$. It can, therefore, be appreciated that both GSCs and their DNA repair mechanisms could be considered as targets for future therapeutic strategies.

TMZ, which is essentially used in conventional chemotherapy for glioblastoma, is a monoalkylating agent that methylates DNA bases. The main cytotoxic lesions induced by $\mathrm{TMZ}$ are $\mathrm{N}_{3}$-methyladenine $\left(\mathrm{N}_{3} \mathrm{MeA}\right), \mathrm{N}_{7^{-}}$ methylguanine $\left(\mathrm{N}_{7}-\mathrm{MeG}\right)$, and $\mathrm{O}_{6}$-methylguanine $\left(\mathrm{O}_{6}-\mathrm{MeG}\right)$ (9). $\mathrm{N}_{3} \mathrm{MeA}$ and $\mathrm{N}_{7}-\mathrm{MeG}$ are repaired by base-excision repair (BER), whereas $\mathrm{O}_{6}-\mathrm{MeG}$ generated by $\mathrm{TMZ}$ is repaired by $\mathrm{O}_{6}$-methylguanine-DNA-methyltransferase (MGMT). Inhibition of either repair pathway can sensitize glioblastoma cells to TMZ cytotoxicity, but BER is naturally potent in GSCs; thus, it contributes minimally to overall TMZ responsiveness. In contrast, MGMT expression has been suppressed by promoter methylation in approximately $40 \%$ of glioblastomas; thus, the amount of MGMT expressed in tumor cells determines whether the tumor cells are susceptible or resistant to $\operatorname{TMZ}(9,10)$. In tumors that show insufficient MGMT expression, persistent $\mathrm{O}_{6}-\mathrm{MeG}$ lesions are incorrectly paired with thymidine and trigger replicative stress of mismatch repair (MMR), resulting in replication fork-associated DNA double-strand breaks (DSBs) and cytotoxicity (11). On the other hand, in patients who have tumors with an abundant expression of MGMT, even TMZ could not improve their prognosis $(9,11)$.

Poly (ADP-ribose) polymerase (PARP) is an important family of enzymes that contribute to repairing DNA damage, achieving genomic stability of tumor cells to promote survival $(7,9,11)$. Therefore, inhibiting PARP has been considered to enhance the potency of cytotoxic agents. PARP inhibitors in combination with TMZ were first used in clinical trials against advanced tumors in 2003 (12). Since the introduction of the concept of synthetic lethality by Bryant et al. (13) and Farmer et al. (14) in 2005, which appeared in homologous recombination-deficient cells exposed to PARP inhibitors, there is a large number of studies on PARP inhibitors as potential antitumor sensitizing agents $(7,11)$. Therefore, several preclinical and clinical studies have confirmed the effect of PARP inhibitors in BRCA-mutant tumors, such as hereditary breast and ovarian cancer (12). Similarly, the efficacy of PARP inhibitors as potent radiosensitizers has also been investigated in gliomas several times $(12,15)$. Despite this, there is not enough evidence to demonstrate that combination therapy with PARP inhibitors and TMZ for treating glioblastoma merits a clinical trial.

In this study, we evaluated whether combination therapy of the PARP inhibitor olaparib with the alkylating agent temozolomide could be a promising treatment for glioblastoma in preclinical models. We tested cell viability and used an orthotropic xenograft model to determine the efficacy of the treatment.

\section{Materials and Methods}

Cell culture. The human glioma cell lines used in the studies were selected based on their MGMT expression, which is related to temozolomide sensitivity. The MGMT promoter-methylated cell lines (U87MG, U251MG) and the MGMT promoter-unmethylated cell line (T98G) were all purchased from the American Type Culture Collection (ATCC, Manassas, VA, UAS) in 2019. Cells were cultured in complete media with Dulbecco's modified Eagle's medium (DMEM, Sigma-Aldrich, Germany) supplemented with $10 \%$ heat-inactivated fetal bovine serum (FBS) and incubated at $37^{\circ} \mathrm{C}$ and $5 \% \mathrm{CO}_{2}$. We used differentiated cell cultures (FBSsupported monolayers) instead of undifferentiated cell cultures (neurospheres) based on a previous study, which found that there were no significant differences between the two cell culture systems in TMZ-induced DNA damage (16).

Cell viability test. We conducted cytotoxicity tests of olaparib. All cells (U87MG, U251MG, T98G) were seeded in 96-well plates $\left(5 \times 10^{3}\right.$ cells/well $)$ and incubated for $24 \mathrm{~h}$. The media used during incubation were discarded and replaced with $200 \mu$ of complete media mixture containing serial increasing concentrations of olaparib (1 nM, $10 \mathrm{nM}, 100 \mathrm{nM}, 1 \mu \mathrm{M}, 10 \mu \mathrm{M}, 100 \mu \mathrm{M}$, and $1 \mathrm{mM}$ ). The cytotoxicity of olaparib, TMZ and a combination of olaparib and TMZ was measured in each cell line. The concentration of TMZ was chosen experientially after several tests to determine the proper concentration that showed a definite inhibition (minimum inhibitory concentration). All groups of cells were plated first in triplicate and then in quadruplicate for statistical analysis.

Cell viability was tested using CCK-8 assays (Dojindo Molecular Technologies, Inc., Tokyo, Japan) according to the manufacturer's protocol. We replaced the treatment solution with $200 \mu \mathrm{l}$ of complete media mixture containing $10 \mu \mathrm{l}$ of CCK-8 solution in each well and incubated for $2 \mathrm{~h}$. CCK- 8 absorbance was then measured using an ELISA reader (VERSAmax microplate reader, Molecular Devices, San Jose, CA, USA) at $450 \mathrm{~nm}$. The viability of the tumor cells was assessed by calculating the Optical Density (OD) ratio of the specific OD in each sample to the one of the control sample. The data were analyzed using SoftMax Pro software (Molecular Devices).

Apoptosis analysis. The apoptosis assay was carried out using the FITC Annexin V Apoptosis Detection Kit I (BD Pharmingen ${ }^{\mathrm{TM}}$, Cat. 556547) according to the manufacturer's protocol. U87MG cells $\left(1 \times 10^{6}\right)$ were seeded in seven $100 \mathrm{~mm}$ petri dishes and incubated in complete media for $24 \mathrm{~h}$. Further incubation was carried out for 48 $\mathrm{h}$ after replacing the media with the following: i) serum-free DMEM (four dishes, control groups), ii) $500 \mu \mathrm{M} \mathrm{TMZ}$ in serum-free DMEM (TMZ group), iii) $10 \mu \mathrm{M}$ olaparib in serum-free DMEM (Ola group) and iv) $500 \mu \mathrm{M} \mathrm{TMZ}+10 \mu \mathrm{M}$ olaparib in serum-free DMEM (TMZ+Ola group). Subsequently, the cells were harvested 
and incubated with $5 \mu \mathrm{l}$ of FITC Annexin V and $5 \mu \mathrm{l}$ PI for 15 min in the dark at RT. The number of apoptotic cells was evaluated by manually counting four random microscopic fields (400x original magnification) per subject. Cells were analyzed using a FACSCanto II (Becton Dickinson) flow cytometer.

Mouse glioblastoma xenograft model. This study was approved by the Institutional Animal Care and Use Committee of the Medical Science Research Institute, Seoul National University Bundang Hospital (Gyeonggi-do, Republic of Korea; authorization No. BA1906-274-033-08). Twenty-two six-week-old BALB/c nude mice were used for the orthotopic xenograft model (Orient Bio, Seongnam-si, Korea; distributor for Charles River, Wilmington, MA, USA). After anesthetizing them by $30 \mathrm{mg} / \mathrm{kg} /$ intraperitoneal(i.p.) Zoletil and $10 \mathrm{mg} / \mathrm{kg} / \mathrm{i} . p$. Rompun, we used a stereotactic frame (Kopf Instruments, Tujunga, CA) to fix them during implantation. Using a $10 \mu \mathrm{l}$ Hamilton syringe, we injected $2 \times 10^{5}$ U87MG cells resuspended in $2 \mu \mathrm{l}$ of Dulbecco's phosphate buffered solution (PBS) intracranially at an identical site $(0.5 \mathrm{~mm}$ anterior and $2.0 \mathrm{~mm}$ right lateral from bregma). Injection lasted for $4 \mathrm{~min}(0.5 \mu \mathrm{l} / \mathrm{min})$, and the needle was removed after exactly $4 \mathrm{~min}$ at a speed of $1 \mathrm{~mm} / \mathrm{min}$. The hole made after the injection in the skull was blocked by dental cement (Prime Dental Manufacturing, Chicago, IL, USA).

Treatment Preparation. The weight/volume percentage concentration of the treatment solutions was $10 \%$. A $10 \%$ hydroxypropyl- $\beta$ cyclodextrin (HBC) solution was prepared with $0.1 \mathrm{~g} \mathrm{HBC}$ powder/10 ml of PBS. First, we vortexed a solution of $100 \mathrm{mg}$ of TMZ powder in $17 \mathrm{ml}$ of $10 \%$ HBC solution, and then a $3 \mathrm{ml}$ solution of dimethyl sulfoxide (DMSO) was then vortexed to optimally solubilize TMZ. Olaparib solution was prepared in the reverse order with the $10 \% \mathrm{HBC}$ solution being vortexed after DMSO was added. This procedure ensured that the substrates were sufficiently dissolved. The prepared treatment solutions were then aliquoted in PCR tubes and cryopreserved for later use. When required, the olaparib and TMZ solutions were thawed for two min in a $37^{\circ} \mathrm{C}$ water bath before the i.p. injection.

Treatment protocol and evaluation. Tumor-bearing mice were randomly allocated to four groups and treated differently after a week of implantation: i) untreated (control group, $n=6$ ), ii) TMZ alone (TMZ group, $n=6$ ), iii) olaparib alone (Ola group, $n=5$ ), and iv) combination with olaparib and TMZ (combination group, $n=5$ ). The dosages for olaparib and TMZ were $50 \mathrm{mg} / \mathrm{kg}$. The mice were received an i.p. injection for 4 consecutive days per week, and the treatment cycle was conducted for 4 weeks. Then, the animals were monitored for 2 months to analyze their survival before sacrifice and measurement of the tumor volume. The humane endpoint was defined as a weight reduction of $>20 \%$ of the initial weight. If weight loss in all mice remained above this threshold, all surviving mice were anaesthetized and humanely sacrificed at the end of the study. Following sacrifice, the brains of the mice were removed following vascular perfusion with a solution containing PBS and fixed with $4 \%$ formaldehyde for 1 week. Then, the brains were embedded in paraffin and sectioned coronally into slices of $5 \mu \mathrm{m}$ thickness using a microtome (RM2255, Leica Biosystems, Richmond, IL, USA). The slices were mounted on individual slides and stained with hematoxylin and eosin. The maximal length (L), width $(\mathrm{W})$, and height $(\mathrm{H})$ of each tumor were measured, and tumor volume was calculated using the following formula: tumor volume $(\mathrm{V})=4 / 3 \pi(\mathrm{L} / 2 \times \mathrm{W} / 2 \times \mathrm{H} / 2)$.
Statistics. Statistics between groups were performed using $t$-tests. The results with a $p$-Value $<0.05$ were considered statistically significant. The Kaplan-Meier method was used for the survival analysis of the experimental animals. Differences with regard to survival were tested for significance using the two-sided log-rank test. All analyses were performed using the SPSS statistical software package (version 21.0; SPSS, Inc., Chicago, IL, USA).

\section{Results}

Cell viability test of olaparib. Since the cytotoxicity of olaparib is unknown in glioma cell lines, it was initially assessed by the cell viability test. The half maximal inhibitory concentration (IC50) of olaparib seemed similar in all three cell lines and was between 0.1 and $1 \mathrm{~mol} / \mathrm{l}$ : i) $228 \mathrm{~mol} / 1 \mathrm{for}$ $\mathrm{U} 87 \mathrm{MG}$, ii) $177 \mathrm{~mol} / 1$ for $\mathrm{U} 251 \mathrm{MG}$, and iii) $260 \mathrm{~mol} / 1$ for T98G. The IC50 values of the three cell lines were similar in terms of olaparib toxicity regardless of the MGMT promoter methylation, and the viability patterns overlapped (Figure 1).

The efficiency of the PARP inhibitor olaparib to sensitize cells to TMZ and help suppress glioblastoma was evaluated in a cell viability test. We found that the degree of viability decreased in the order of i) control, ii) olaparib, iii) temozolomide, and iv) their combination (Figure 2A-C). Olaparib enhanced the TMZ-induced cytotoxicity in all glioblastoma models regardless of the MGMT promoter methylation status. In addition, the difference in cell viability between the Ola and the combination group in T98G, the MGMT promoter-unmethylated cell line, was larger compared to the other two groups (Figure 2C).

Apoptosis analysis. Apoptosis is a natural course of DNAdamaged cells. Since TMZ is a monoalkylating agent that affects DNA, apoptosis instead of necrosis should occur after treatment. Our FACS data showed that after $48 \mathrm{~h}$ of TMZ and olaparib treatment, there were few cells that underwent necrosis $(0.93 \%$ of 10,000 cells in the $\mathrm{TMZ}+\mathrm{Ola}$ group), while a considerable number of cells underwent apoptosis (early apoptosis $3.69 \%$ vs. late apoptosis $12.24 \%$ in the TMZ+Ola group) (Figure 3A). Comparing the $3 \mathrm{~h}$ to the $48 \mathrm{~h}$ treatment group, we found that there were more cells undergoing early apoptosis in the first group (3 h, 7.61\%, 48 $\mathrm{h}, 3.69 \%$ ) but fewer cells were fully destroyed ( $3 \mathrm{~h} 3.88 \%$,

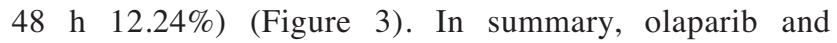
temozolomide cotreatment led glioblastoma tumor cells to undergo apoptosis rather than necrosis.

Cotreatment efficacy in vivo. An orthotopic xenograft model was used to identify the efficacy of PARP inhibitors in sensitizing TMZ to mimic the clinical situation. Animals were treated for 4 weeks with TMZ or PARP inhibitor or both. The inhibitory effects on tumor growth were evaluated by tumor volume assessment. We obtained the mean tumor volume from each group, including the untreated control 


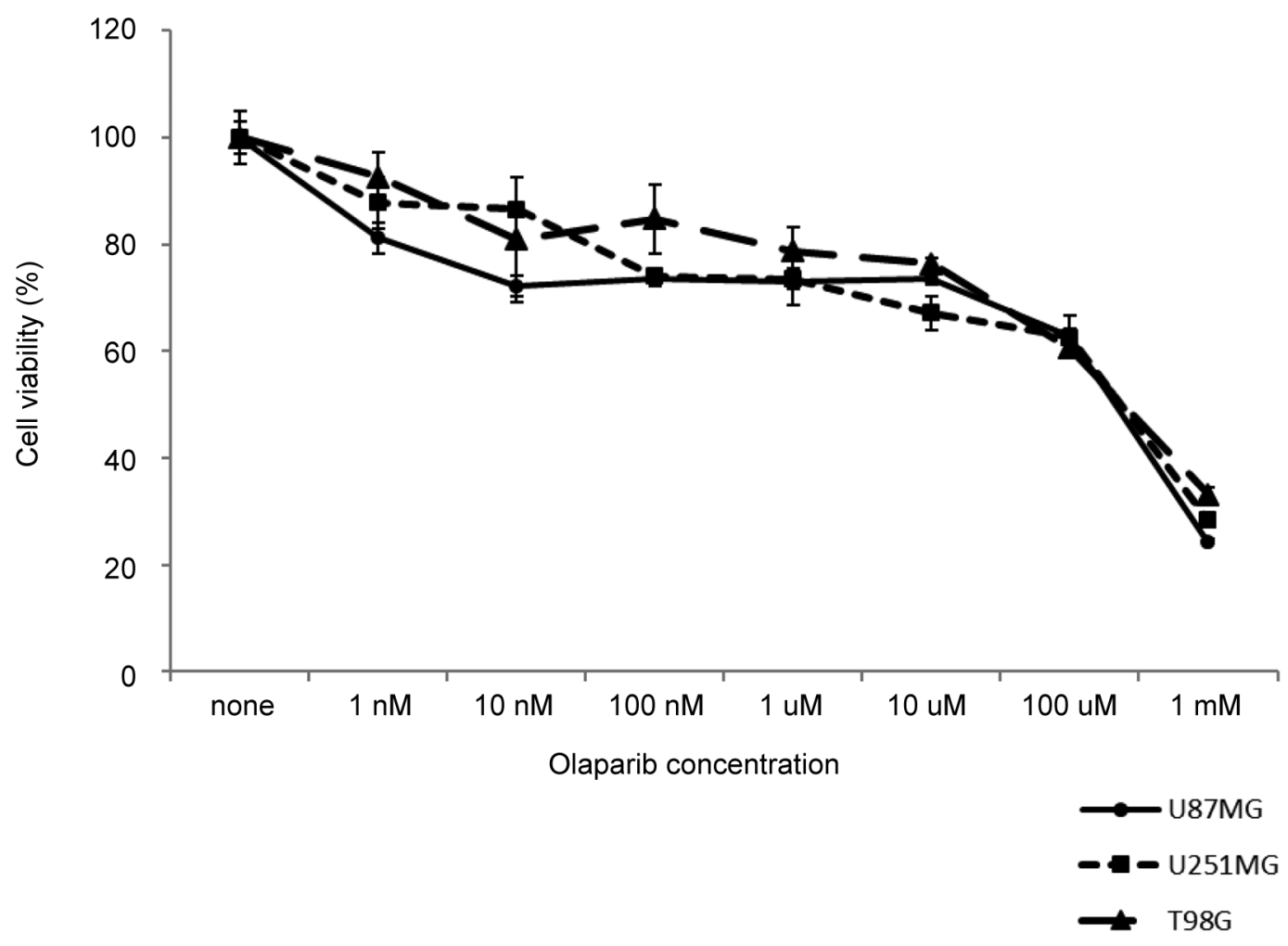

Figure 1. Cell viability of glioma cell lines in olaparib. The half maximal inhibitory concentration (IC50) of olaparib seemed similar in all three cell lines: $228 \mathrm{~mol} / \mathrm{l}$ for U87MG, $177 \mathrm{~mol} / \mathrm{l}$ for U251MG, and $260 \mathrm{~mol} / \mathrm{l}$ for T98G. The overall cell viability pattern was similar within the three glioblastoma cell lines, regardless of the MGMT status. MGMT: $O_{6}$-methylguanine-DNA-methyltransferase.

animals. The mean tumor volume in the TMZ $(14.02 \pm 5.53$ $\left.\mathrm{mm}^{3}\right)$ and the combination group $\left(13.35 \pm 7.03 \mathrm{~mm}^{3}\right)$ was significantly smaller than both the control $\left(45.20 \pm 7.67 \mathrm{~mm}^{3}\right)$ and the Ola group $\left(45.06 \pm 3.88 \mathrm{~mm}^{3}\right)$. The mean tumor volume in the combination group was a bit smaller than that of the TMZ group, however, there was no statistical significance $(p=0.865)$ (Figure $4 \mathrm{~A}$ and $\mathrm{B})$.

Figure 4C shows the survival analysis on the orthotopic mouse model. The mean survival period of the i) control, ii) Ola, iii) TMZ, and iv) combination group was: i) 55, ii) 52, iii) 77 , and iv) 77 days, respectively. The overall survival in both the TMZ $(p=0.018)$ and the combination group ( $p=0.030$ ) was significantly high compared to the control group. There was no significant difference between the TMZ and combination groups.

\section{Discussion}

Olaparib enhanced the cytotoxicity of TMZ on glioblastoma in our in vitro studies. All the glioblastoma cell lines tested were more vulnerable to mixed conditions of TMZ and olaparib than TMZ alone. However, the orthotopic glioblastoma mouse model treated with mixed therapy had no evidence of a survival gain compared to the one offered by TMZ monotherapy. This result shows that combination therapy with a PARP inhibitor and TMZ has promising effects even on glioblastoma, as previous studies have proved its efficiency on BRCA-mutant tumors (12), still with some hurdles in in vivo studies and real clinical settings.

The combined effect of PARP inhibitors and TMZ could be understood at a molecular level. As mentioned above, the resistance of glioblastoma stem cells to TMZ arises from their potent repair system, represented by BER and MGMT. Several components are needed to activate their repair systems, such as PARPs. PARP1, which can be inhibited by olaparib, detects the presence of DNA lesions and then activates signaling pathways to promote relevant cell responses for DNA repair, including BER. When DNA single-strand breaks (SSBs) are detected, they recruit and activate several BER factors as a necessary scaffold to repair SSBs before replication $(17,18)$. Thus, inhibiting PARP1 could prevent GSCs from activating the repair system, making them vulnerable to temozolomide.

In 2015, Erice et al. (19) reported that MGMT expression predicts PARP-mediated resistance to temozolomide. The study verified the sensitizing effect of PARP inhibitors in 

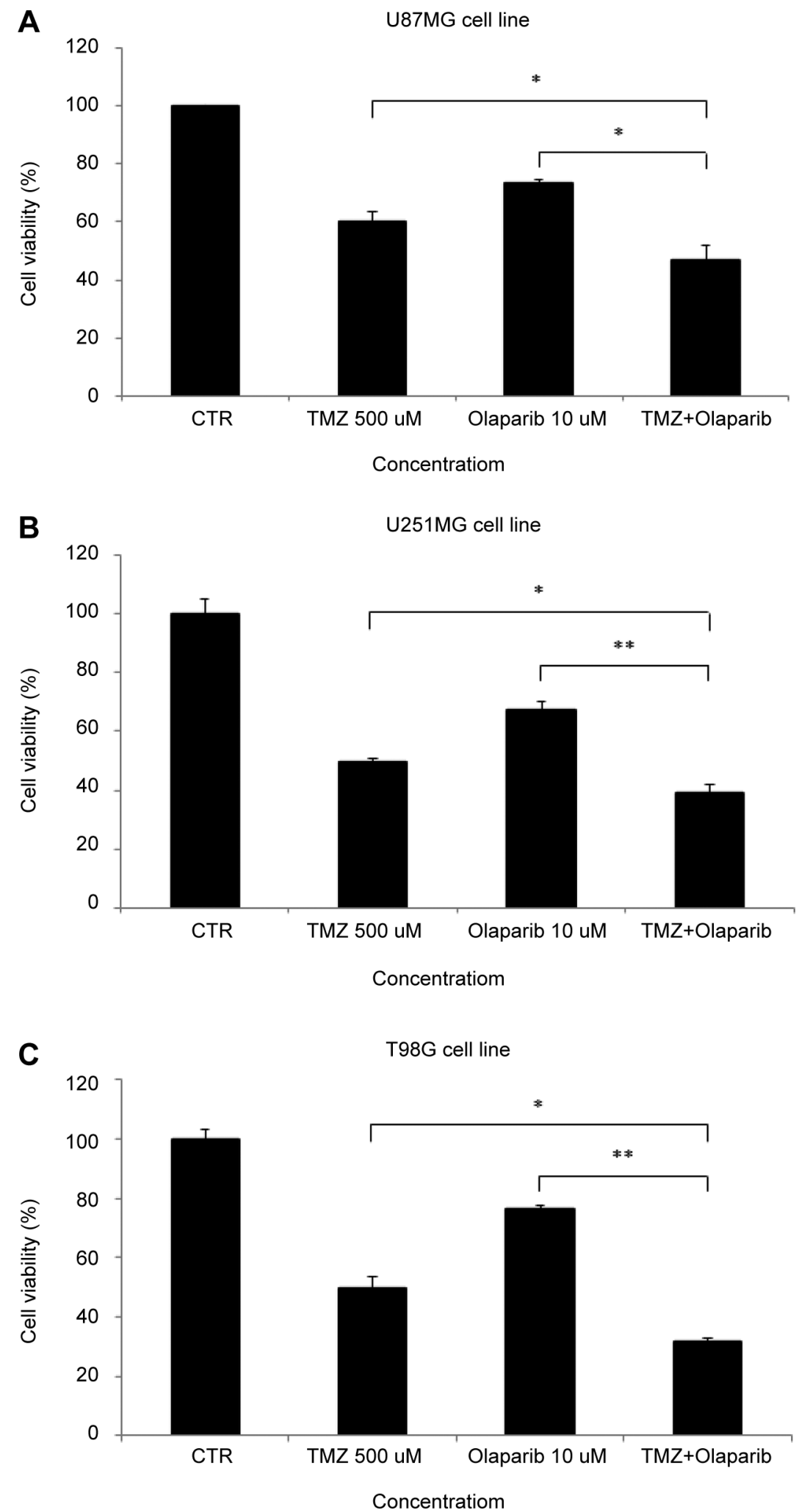

Figure 2. The anti-tumor effect of olaparib, temozolomide, or their combination on the viability of the different cell lines. These cell lines (A; U87MG, $B ; U 251 M G, C ; T 98 G$ ) were seeded in 96-well-plates and treated with olaparib only, temozolomide only, or a combination of the two. Olaparib enhanced the TMZ-induced cytotoxicity in all cell lines. CTR: Control; TMZ: temozolomide. ${ }^{*} p<0.05$, and $* * p<0.001$. 
A

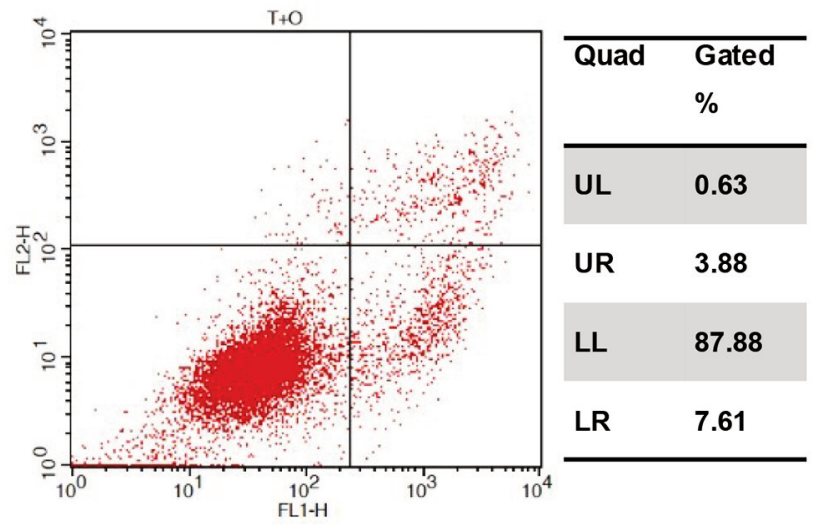

B

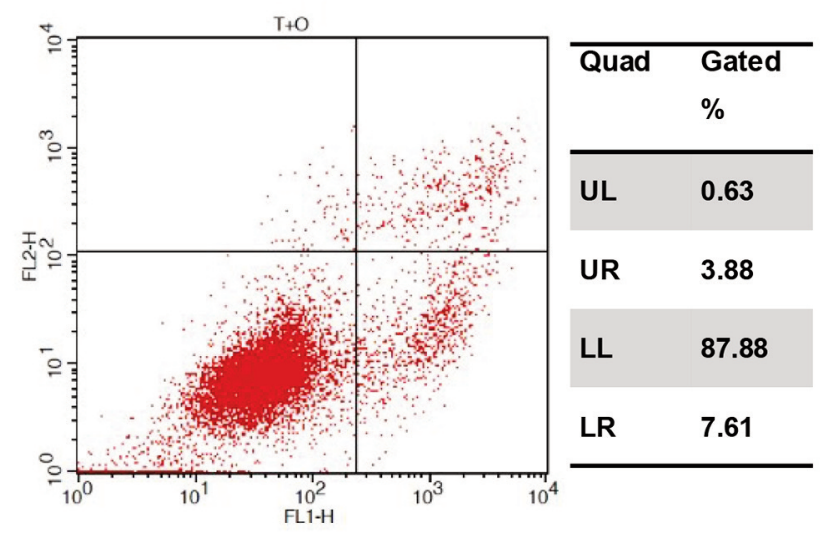

Figure 3. Apoptosis assay of combination treatment in U87MG cell line. FACS data $(A ; 48 h, B ; 3 h)$ of Annexin V/PI apoptosis analysis showed that using Olaparib $(O)$ and Temozolomide $(T)$ induces apoptosis, not necrosis. UL: Upper left; UR: upper right; LL: lower left LR: lower right.

temozolomide-resistant cell lines, especially in the most commonly temozolomide-treated tumors (melanoma, colorectal cancer, and glioblastoma cancer). In their study, PARP inhibitors showed sensitizing effects only in MGMToverexpressing cell lines, which are TMZ-resistant. The authors noted that cell lines that do not express MGMT by promoter methylation did not change their response following the addition of a PARP inhibitor to temozolomide therapy.

According to a previous paper of Erice et al. (19), we assessed the viability of each cell line used in that study to the combination therapy before implanting them into the xenograft model. We found that both MGMT promotermethylated as well as -unmethylated cells were sensitive to the treatment of PARP inhibitors with TMZ. As cell line of MGMT promoter methylation status does not matter, we generated an orthotopic xenograft model by U87MG as previously described (20).
MGMT is a "suicide" DNA repair enzyme whose level is important to maintain genomic stability (21). When MGMT is overexpressed, DNA alkylation formed by chemotherapeutics could be easily repaired, resulting in high resistance (10). However, because PARP inhibitors participate in different DNA repair pathways, base excision repair could make MGMT-overexpressing cell lines vulnerable to temozolomide. This is a great advantage of PARP inhibitors against glioblastoma; however, it does not mean that PARP inhibitors are only effective in MGMT promoter-unmethylated-derived glioblastoma. Given our results, a PARP inhibitor can also enhance the effect of TMZ in MGMT promoter-methylatedderived glioblastoma, which is another advantage of PARP inhibitor use against glioblastoma.

The difference in the coverage of PARP inhibitors between other and our studies could be hidden by proportional statistics. In Erice's study (19), the researchers used half maximal growth inhibition concentration (GI50) as a sensitization marker. This marker could proportionally evaluate how the susceptibility of a cell line changed by a sensitizer. Since GI50 is the concentration for half the maximal inhibition of cell proliferation, this value could rise substantially for agents to which there is resistance, resulting in a significant difference between sensitized and nonsensitized lines. Importantly, in already sensitive lines, this method could underestimate the effect of the agent, although there could be a significant enhancement of reactivity. In our study, we compared the viability of different cell lines under the same temozolomide concentration. This procedure has the benefit that this fixed dose of TMZ, since efficient, could be used for glioblastoma patients. In addition, analysis of cell viability showed that a sufficient amount of PARP inhibitor could enhance the cytotoxicity of TMZ.

Interestingly, against our expectations, we failed to demonstrate the survival gain of olaparib and TMZ combination treatment compared to TMZ monotherapy in the in vivo study. This could be partly explained based on the assumption of an ineffective drug penetration into the brain due to the blood-brain barrier. According to Parrish et al., the PARP inhibitor rucaparib does not potentiate TMZ in the orthotopic GBM models (22), which does not agree with the findings of an in vivo study in flank tumor models (23). Blood-brain barrier remains a hurdle to most chemotherapeutics in tumors of the central nervous system, and olaparib is not an exception. Besides, we could not evaluate the tumor model before treatment. Despite the fact that each treatment group showed no difference in mouse weight or general health status, there might have been a difference in tumor volume, which can affect the efficacy of drugs.

Recently, two phase I trials use olaparib in glioblastoma patients and their results appear to be promising. In the first trial olaparib combined with radiotherapy was well tolerated in newly diagnosed glioblastoma patients (24). In the second 
A

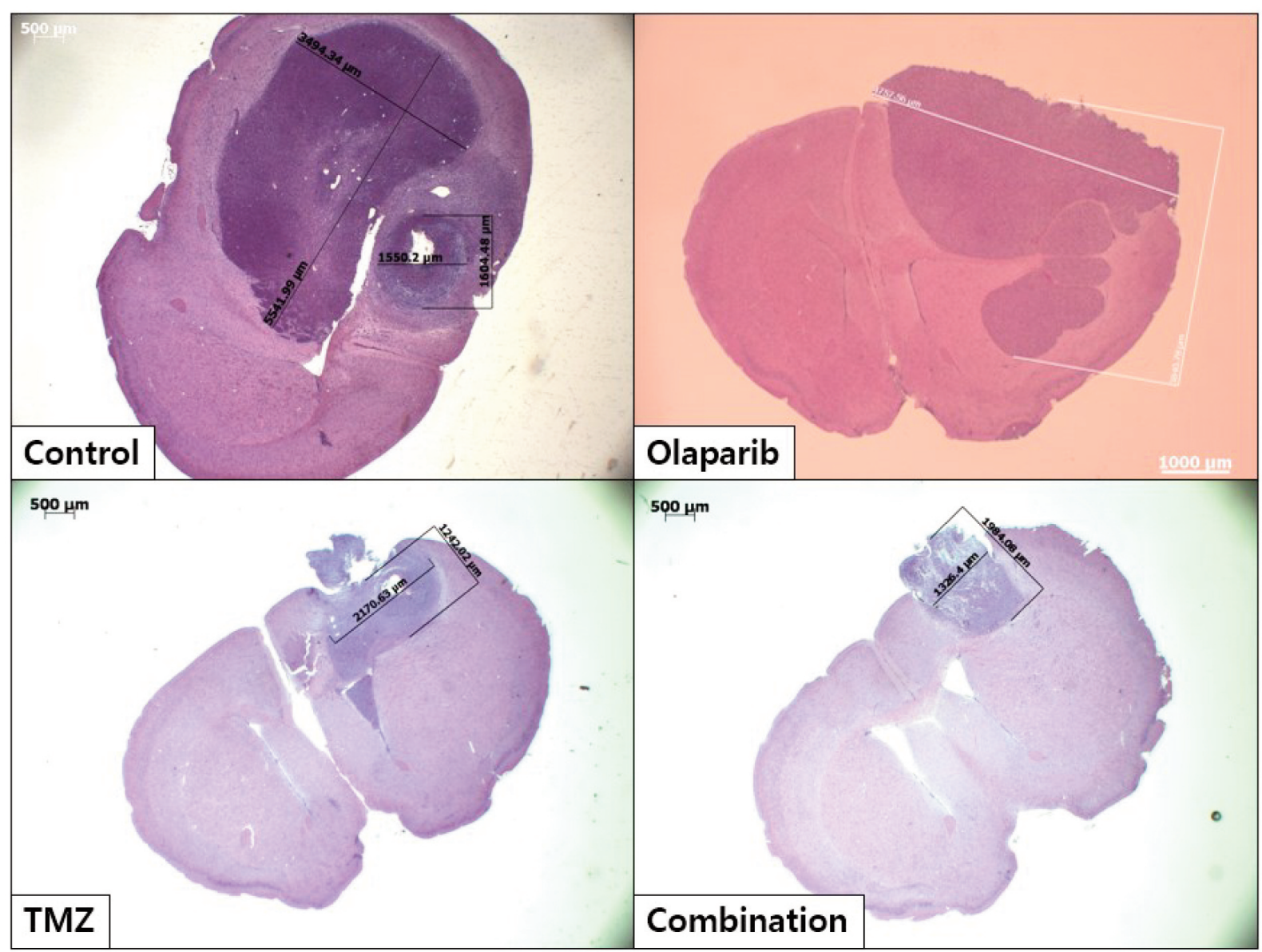

B

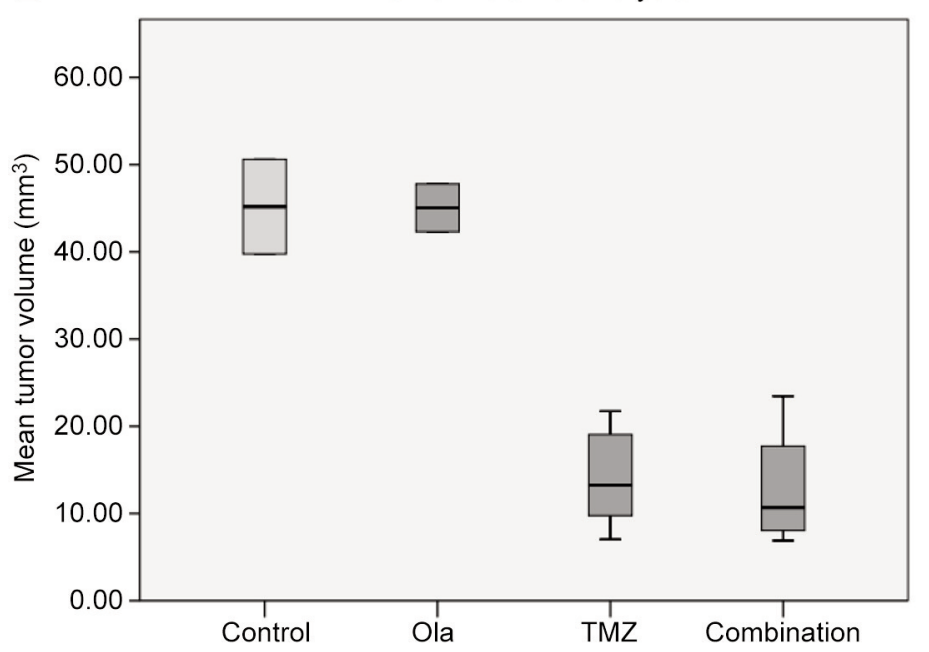

C Survival analysis

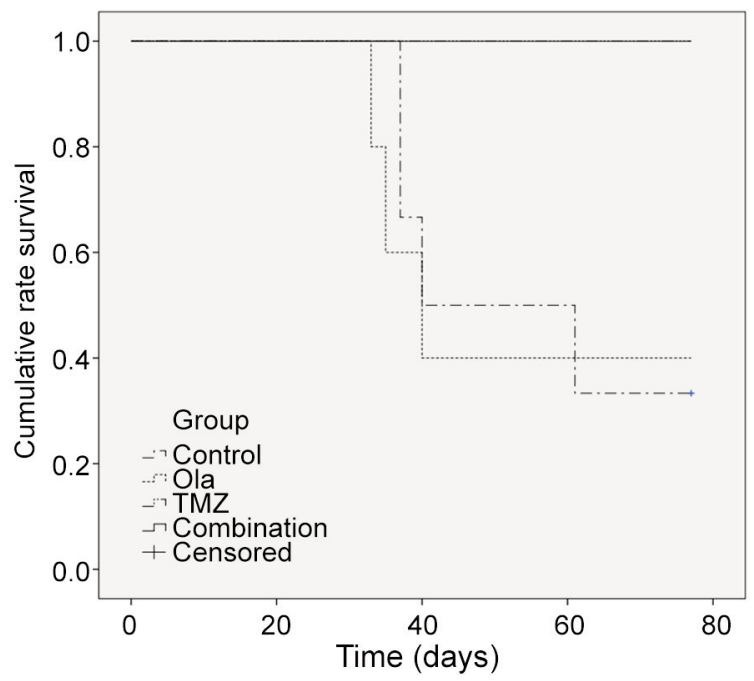

Figure 4. The tumor volume and survival in the glioblastoma xenograft model. (A) Hematoxylin and eosin-stained coronal histological section of mouse brain of each group analyzed 1 month after treatment. (B) The mean tumor volume of the TMZ group and the combination treatment group was significantly lower than the control group, however, between them, there was no statistical significance. (C) Survival of the glioma xenograft mouse model. Most of the control group and the Olaparib (Ola) group mice died within 40 days. However, mice in the remaining groups lived to the end of the experiment. In addition, there was no difference between the TMZ group and the combination group. TMZ: Temozolomide. 
trial, olaparib and temozolomide combination therapy was conducted in patients with relapsed glioblastoma, and the researchers found that olaparib penetrates the core and margins of recurrent glioblastoma, with good tolerance (25).

Only a handful of studies have assessed the use of PARP inhibitors in glioblastoma. In 2017, Ohmoto et al. (26) indicated four research tasks needed to apply PARP inhibitors in specific tumor treatments. First, the most suitable drugs should be determined among several PARP inhibitors. Second, a new treatment target should be found in cases of homologous recombination repair (HHR)-proficient tumors. Third, the regimen that could increase the overall survival and progression-free survival should be decided, and fourth, proper biomarkers that could measure the efficacy, side effects, and clinical resistance should be found.

Our study has some limitations. First, we could not evaluate the penetration or accumulation of olaparib or temozolomide, which would affect the efficacy of treatment. Many other studies have noted the limited role of olaparib due to the blood brain barrier, limiting the exact role of novel drugs. Second, the initial tumor volume was not considered due to technical limitations. The initial tumor volume could confound the interpretation of the results.

Additional studies with different cell lines or other PARP inhibitors and further evaluation of drug distribution in brain tumors would be beneficial to determine the effect of PARP inhibitors in patients with glioblastoma.

\section{Conflicts of Interest}

The Authors have no conflicts of interest to disclose.

\section{Authors' Contributions}

$\mathrm{KH}, \mathrm{JHH}$ and CYK designed the study and interpreted the results. $\mathrm{KH}, \mathrm{JHL}, \mathrm{SHK}$ and KOG performed the experiments and analyzed data. SYJ provided the technical support. KH and JHL wrote the manuscript. All Authors have approved the publication of the study.

\section{Acknowledgements}

This research was supported by a grant from the Korea Health Technology R\&D Project through the Korea Health Industry Development Institute (KHIDI), funded by the Ministry of Health \& Welfare, Republic of Korea (grant number: HI19C0583) and by grants from the Seoul National University Bundang Hospital Research Fund (grant nos. 14-2014-008).

\section{References}

1 Ostrom QT, Bauchet L, Davis FG, Deltour I, Fisher JL, Langer CE, Pekmezci M, Schwartzbaum JA, Turner MC, Walsh KM, Wrensch MR and Barnholtz-Sloan JS: The epidemiology of glioma in adults: a "state of the science" review. Neuro Oncol 16(7): 896-913, 2014. PMID: 24842956. DOI: 10.1093/neuonc/ nou 087
2 Stupp R, Mason WP, van den Bent MJ, Weller M, Fisher B, Taphoorn MJ, Belanger K, Brandes AA, Marosi C, Bogdahn U, Curschmann J, Janzer RC, Ludwin SK, Gorlia T, Allgeier A, Lacombe D, Cairncross JG, Eisenhauer E, Mirimanoff RO, European Organisation for Research and Treatment of Cancer Brain Tumor and Radiotherapy Groups and National Cancer Institute of Canada Clinical Trials Group: Radiotherapy plus concomitant and adjuvant temozolomide for glioblastoma. N Engl J Med 352(10): 987-996, 2005. PMID: 15758009. DOI: 10.1056/NEJMoa043330

3 Ostrom QT, Cioffi G, Gittleman H, Patil N, Waite K, Kruchko $\mathrm{C}$ and Barnholtz-Sloan JS: CBTRUS statistical report: primary brain and other central nervous system tumors diagnosed in the United States in 2012-2016. Neuro Oncol 21(Suppl 5): v1-v100, 2019. PMID: 31675094. DOI: 10.1093/neuonc/noz150

4 Minniti G, Amelio D, Amichetti M, Salvati M, Muni R, Bozzao A, Lanzetta G, Scarpino S, Arcella A and Enrici RM: Patterns of failure and comparison of different target volume delineations in patients with glioblastoma treated with conformal radiotherapy plus concomitant and adjuvant temozolomide. Radiother Oncol 97(3): 377-381, 2010. PMID: 20855119. DOI: 10.1016/j.radonc. 2010.08 .020

5 Lawson HC, Sampath P, Bohan E, Park MC, Hussain N, Olivi A, Weingart $\mathrm{J}$, Kleinberg $\mathrm{L}$ and Brem $\mathrm{H}$ : Interstitial chemotherapy for malignant gliomas: the Johns Hopkins experience. J Neurooncol 83(1): 61-70, 2007. PMID: 17171441. DOI: $10.1007 / \mathrm{s} 11060-006-9303-1$

6 Liu Y, Shen Y, Sun T and Yang W: Mechanisms regulating radiosensitivity of glioma stem cells. Neoplasma 64(5): 655-665, 2017. PMID: 28592117. DOI: 10.4149/neo_2017_502

7 Murat A, Migliavacca E, Gorlia T, Lambiv WL, Shay T, Hamou MF, de Tribolet N, Regli L, Wick W, Kouwenhoven MC, Hainfellner JA, Heppner FL, Dietrich PY, Zimmer Y, Cairncross JG, Janzer RC, Domany E, Delorenzi M, Stupp R and Hegi ME: Stem cell-related "self-renewal" signature and high epidermal growth factor receptor expression associated with resistance to concomitant chemoradiotherapy in glioblastoma. J Clin Oncol 26(18): 3015-3024, 2008. PMID: 18565887. DOI: 10.1200/JCO. 2007.15.7164

8 Ahmed SU, Carruthers R, Gilmour L, Yildirim S, Watts C and Chalmers AJ: Selective inhibition of parallel DNA damage response pathways optimizes radiosensitization of glioblastoma stem-like cells. Cancer Res 75(20): 4416-4428, 2015. PMID: 26282173. DOI: 10.1158/0008-5472.CAN-14-3790

9 Sarkaria JN, Kitange GJ, James CD, Plummer R, Calvert H, Weller $\mathrm{M}$ and Wick W: Mechanisms of chemoresistance to alkylating agents in malignant glioma. Clin Cancer Res 14(10): 2900-2908, 2008. PMID: 18483356. DOI: 10.1158/10780432.CCR-07-1719

10 Hegi ME, Diserens AC, Gorlia T, Hamou MF, de Tribolet N, Weller M, Kros JM, Hainfellner JA, Mason W, Mariani L, Bromberg JE, Hau P, Mirimanoff RO, Cairncross JG, Janzer RC and Stupp R: MGMT gene silencing and benefit from temozolomide in glioblastoma. N Engl J Med 352(10): 9971003, 2005. PMID: 15758010. DOI: 10.1056/NEJMoa043331

11 D'Atri S, Tentori L, Lacal PM, Graziani G, Pagani E, Benincasa E, Zambruno G, Bonmassar E and Jiricny J: Involvement of the mismatch repair system in temozolomide-induced apoptosis. Mol Pharmacol 54(2): 334-341, 1998. PMID: 9687575. DOI: $10.1124 / \mathrm{mol} .54 .2 .334$ 
12 Lesueur P, Chevalier F, Austry JB, Waissi W, Burckel H, Noël G, Habrand JL, Saintigny Y and Joly F: Poly-(ADP-ribose)polymerase inhibitors as radiosensitizers: a systematic review of pre-clinical and clinical human studies. Oncotarget 8(40): 6910569124, 2017. PMID: 28978184. DOI: 10.18632/oncotarget. 19079

13 Bryant HE, Schultz N, Thomas HD, Parker KM, Flower D, Lopez E, Kyle S, Meuth M, Curtin NJ and Helleday T: Specific killing of BRCA2-deficient tumours with inhibitors of poly(ADP-ribose) polymerase. Nature 434(7035): 913-917, 2005. PMID: 15829966. DOI: 10.1038/nature03443

14 Farmer H, McCabe N, Lord CJ, Tutt AN, Johnson DA, Richardson TB, Santarosa M, Dillon KJ, Hickson I, Knights C, Martin NM, Jackson SP, Smith GC and Ashworth A: Targeting the DNA repair defect in BRCA mutant cells as a therapeutic strategy. Nature 434(7035): 917-921, 2005. PMID: 15829967. DOI: $10.1038 /$ nature 03445

15 Lesueur P, Chevalier F, El-Habr EA, Junier MP, Chneiweiss H, Castera L, Müller E, Stefan D and Saintigny Y: Radiosensitization effect of talazoparib, a Parp inhibitor, on glioblastoma stem cells exposed to low and high linear energy transfer radiation. Sci Rep 8(1): 3664, 2018. PMID: 29483558. DOI: $10.1038 / \mathrm{s} 41598-018-22022-4$

16 Gupta SK, Mladek AC, Carlson BL, Boakye-Agyeman F, Bakken KK, Kizilbash SH, Schroeder MA, Reid J and Sarkaria JN: Discordant in vitro and in vivo chemopotentiating effects of the PARP inhibitor veliparib in temozolomide-sensitive versus resistant glioblastoma multiforme xenografts. Clin Cancer Res 20(14): 3730-3741, 2014. PMID: 24838527. DOI: 10.1158/ 1078-0432.CCR-13-3446

17 Gradwohl G, Ménissier de Murcia JM, Molinete M, Simonin F, Koken M, Hoeijmakers JH and de Murcia G: The second zincfinger domain of poly(ADP-ribose) polymerase determines specificity for single-stranded breaks in DNA. Proc Natl Acad Sci U.S.A. 87(8): 2990-2994, 1990. PMID: 2109322. DOI: 10.1073/pnas.87.8.2990

18 Helleday T, Petermann E, Lundin C, Hodgson B and Sharma RA: DNA repair pathways as targets for cancer therapy. Nat Rev Cancer 8(3): 193-204, 2008. PMID: 18256616. DOI: 10.1038/ $\operatorname{nrc} 2342$

19 Erice O, Smith MP, White R, Goicoechea I, Barriuso J, Jones C, Margison GP, Acosta JC, Wellbrock C and Arozarena I: MGMT expression predicts PARP-mediated resistance to temozolomide. Mol Cancer Ther 14(5): 1236-1246, 2015. PMID: 25777962. DOI: $10.1158 / 1535-7163 . M C T-14-0810$

20 Jin J, Hwang K, Joo JD, Han JH and Kim CY: Combination therapy of 7-O-succinyl macrolactin A tromethamine salt and temozolomide against experimental glioblastoma. Oncotarget 9(2): 2140-2147, 2017. PMID: 29416760. DOI: 10.18632/ oncotarget.23295
21 Molenaar RJ, Verbaan D, Lamba S, Zanon C, Jeuken JW, BootsSprenger SH, Wesseling P, Hulsebos TJ, Troost D, van Tilborg AA, Leenstra S, Vandertop WP, Bardelli A, van Noorden CJ and Bleeker FE: The combination of IDH1 mutations and MGMT methylation status predicts survival in glioblastoma better than either IDH1 or MGMT alone. Neuro Oncol 16(9): 1263-1273, 2014. PMID: 24510240. DOI: 10.1093/neuonc/nou005

22 Parrish KE, Cen L, Murray J, Calligaris D, Kizilbash S, Mittapalli RK, Carlson BL, Schroeder MA, Sludden J, Boddy AV, Agar NY, Curtin NJ, Elmquist WF and Sarkaria JN: Efficacy of PARP inhibitor rucaparib in orthotopic glioblastoma xenografts is limited by ineffective drug penetration into the central nervous system. Mol Cancer Ther 14(12): 2735-2743, 2015. PMID: 26438157. DOI: 10.1158/1535-7163.MCT-15-0553

23 Thomas HD, Calabrese CR, Batey MA, Canan S, Hostomsky Z, Kyle S, Maegley KA, Newell DR, Skalitzky D, Wang LZ, Webber SE and Curtin NJ: Preclinical selection of a novel poly(ADP-ribose) polymerase inhibitor for clinical trial. Mol Cancer Ther 6(3): 945-956, 2007. PMID: 17363489. DOI: 10.1158/1535-7163.MCT-06-0552

24 Chalmers A, Short S, Watts C, Herbert C, Morris A, Stobo J, Cruickshank G, Dunn L, Erridge S, Godfrey L, Jefferies S, Lopez J, Mcbain C, Pittman M, Dillon S, James A, Nowicki S, Williamson A, Kelly C and Halford S: Phase I clinical trials evaluating olaparib in combination with radiotherapy (RT) and/or temozolomide (TMZ) in glioblastoma patients: Results of OPARATIC and PARADIGM phase I and early results of PARADIGM-2. Journal of Clinical Oncology 36(15_suppl): 2018-2018, 2019. DOI: 10.1200/JCO.2018.36.15_suppl.2018

25 Halford S, Cruickshank G, Dunn L, Erridge S, Godfrey L, Herbert C, Jefferies S, Lopez J, Mcbain C, Pittman M, Sleigh R, Watts C, Webster-smith $\mathrm{M}$ and Chalmers A: Results of the OPARATIC trial: A phase I dose escalation study of olaparib in combination with temozolomide (TMZ) in patients with relapsed glioblastoma (GBM). Journal of Clinical Oncology 35(15_suppl): 2022-2022, 2020. DOI: 10.1200/JCO.2017. 35.15_suppl.2022

26 Ohmoto A and Yachida S: Current status of poly(ADP-ribose) polymerase inhibitors and future directions. Onco Targets Ther 10: 5195-5208, 2017. PMID: 29138572. DOI: 10.2147/ OTT.S139336
Received March 23, 2021

Revised April 17, 2021

Accepted May 4, 2021 